\title{
Transthoracic echocardiography for imaging of the different coronary artery segments: a feasibility study Johnny Vegsundvåg*1, Espen Holte ${ }^{1}$, Rune Wiseth ${ }^{2,3}$, Knut Hegbom³ and Torstein Hole 1,4 $^{1,4}$
}

Address: ${ }^{1}$ Department of Internal Medicine, Ålesund Hospital, Ålesund, Norway, ${ }^{2}$ Department of Circulation and Medical Imaging, Norwegian University of Science and Technology (NTNU), Trondheim, Norway, ${ }^{3}$ Department of Cardiology, Trondheim University Hospital, Trondheim, Norway and ${ }^{4}$ Medical Faculty, Norwegian University of Science and Technology (NTNU), Trondheim, Norway

Email: Johnny Vegsundvåg* - johnnyvegsundvag@adsl.no; Espen Holte - es-holte@online.no; Rune Wiseth - rune.wiseth@stolav.no; Knut Hegbom - knut.hegbom@stolav.no; Torstein Hole - torstein.hole@helse-sunnmore.no

* Corresponding author

Published: 22 December 2009

Cardiovascular Ultrasound 2009, 7:58 doi:10.1 |86//476-7|20-7-58
Received: 26 August 2009

Accepted: 22 December 2009

This article is available from: http://www.cardiovascularultrasound.com/content/7/I/58

(C) 2009 Vegsundvåg et al; licensee BioMed Central Ltd.

This is an Open Access article distributed under the terms of the Creative Commons Attribution License (http://creativecommons.org/licenses/by/2.0), which permits unrestricted use, distribution, and reproduction in any medium, provided the original work is properly cited.

\begin{abstract}
Background: Transthoracic echocardiography (TTE) may be used for direct inspection of various parts of the main coronary arteries for detection of coronary stenoses and occlusions. We aimed to assess the feasibility of TTE to visualise the complete segments of the left main (LM), left descending (LAD), circumflex $(C x)$ and right (RCA) coronary arteries.
\end{abstract}

Methods: One hundred and eleven patients scheduled for diagnostic coronary angiography because of chest pain or acute coronary syndrome had a TTE study to map the passage of the main coronary arteries. LAD, Cx and RCA were each divided into proximal, middle and distal segments. If any part of the individual segment of a coronary artery with antegrade blood flow was not visualised, the segment was labeled as not satisfactorily seen.

Results: Complete imaging of the LM was achieved in $98 \%$ of the patients. With antegrade directed coronary artery flow, the proximal, middle and distal segments of LAD were completely seen in $96 \%, 95 \%$ and $91 \%$ of patients, respectively. Adding the completely seen segments with antegrade coronary flow and segments with retrograde coronary flow, the proximal, middle and distal segments of LAD were adequately visualised in $96 \%, 96 \%$ and $93 \%$ of patients, respectively. With antegrade directed coronary artery flow, the proximal, middle and distal segments of $\mathrm{Cx}$ were completely seen in $88 \%, 61 \%$ and $3 \%$ and in RCA in $40 \%, 28 \%$ and $54 \%$ of patients. Retrograde coronary artery flow was correctly identified as verified by coronary angiography in seven coronary segments, mainly in the posterior descending artery (labeled as the distal segment of RCA) and distal LAD.

Conclusions: TTE is a feasible method for complete demonstration of coronary flow in the LM, the proximal $C x$ and the different segments of LAD, but less suitable for the RCA and mid and distal segments of the Cx. (ClinicalTrials.gov number NTC0028I346.) 


\section{Background}

Non-invasive imaging of coronary arteries by transthoracic echocardiography (TTE) is an emerging diagnostic tool for studying flow in the left main (LM), the left descending artery (LAD), the circumflex $(\mathrm{Cx})$ and the right coronary arteries (RCA) [1,2]. Direct visualisation of segments of the coronary arteries may help in diagnosing significant coronary artery stenoses [2-4]. With this technique, a coronary stenosis typically exhibits local flow acceleration and turbulence expressed as colour aliasing by colour flow Doppler and accelerated flow velocities across the stenosis [2-5]. Total occlusion of a coronary artery may be detected by retrograde flow in the same artery [6-9]. However, demonstration of stenosis or retrograde flow in the main coronary arteries by TTE is dependent on optimal visualisation of the different segments of each main coronary artery. The aim of this study was to assess the feasibility of TTE to visualise the complete segments of the LM, LAD, Cx and RCA in a larger set of patients.

\section{Methods}

\section{Study population}

Patients were consecutively included in the study if they fulfilled the following criteria: (i) already scheduled for diagnostic coronary angiography because of chest pain (typical or atypical angina pectoris), or coronary angiography was planned because of acute coronary syndrome; (ii) patient age above 18 years; (iii) met no exclusion criteria. The exclusion criteria were: (i) previous coronary artery bypass surgery; (ii) presumed insufficient acoustic windows because of severe emphysema or gross overweight; (iii) significant valvular disease; (iv) atrial fibrillation; (v) administrative reasons (logistics).

The study protocol was approved by the Regional Committee for Medical and Health Research Ethics and the Norwegian Data Inspectorate. All participants gave written, informed consent. ClinicalTrials.gov number NTC00281346.

Six patients did not enter the study because of insufficient acoustic windows $(n=3)$, lack of consent $(n=2)$ or aortic stenosis $(n=1)$. We included 115 patients in the study, but 4 patients were later excluded from further analysis because of protocol violation: aortic stenosis $(n=2)$, atrial fibrillation $(\mathrm{n}=2)$. The final study group consisted of 111 patients. Clinical characteristics of the patients are presented in Table 1. All patients took their prescribed medication the day of the echocardiographic study (Table 1).

\section{Echocardiographic evaluation}

Patients were examined with an Acuson Sequoia c 512 (Siemens Medical Solutions Inc, USA) ultrasound system connected to standard $4 \mathrm{~V} 1 \mathrm{C}$ and $7 \mathrm{~V} 3 \mathrm{C}$ transthoracic transducers. The anatomical course of the coronary arteries was examined by use of colour Doppler mapping with data postprocessing mix function, which makes the colours transparent. The velocity scale of colour Doppler was set to $0,24 \mathrm{~m} / \mathrm{s}$, but was actively changed to provide optimal images. Contrast agent was not used.

With the patient in the supine, left and right lateral decubitus positions, all standard and modified apical, parasternal, and subcostal views were used to follow the course of the LM, LAD, Cx and RCA, from the start of each artery and distally as far as possible. With the patient in the left lateral decubitus position, the LM was examined from the left parasternal short- and long-axis views focusing on the area adjacent to the left sinus of Valsalva cranial to the aortic valve (Figure $1 \mathrm{~A}$ ). In the same short- and long-axis views the proximal LAD ( $\mathrm{pLAD}$ ) could be seen leaving the LM and turning slightly towards the transducer (Figure 1A). The LM and pLAD could also be imaged from modified apical 5- and 2-chamber views in many patients. Origin of the first septal branch was often identified, marking the transition from the pLAD to the middle segment of LAD (mLAD) (Figure 1B). If the first septal branch was not visualised, the division between the pLAD and mLAD was set approximately halfway to the level of the left ventricular papillary muscles. The course of the mLAD and distal LAD (dLAD) was imaged from parasternal modified short- and long-axis views focusing on the anterior interventricular sulcus, and the same arterial segments could also be seen from modified apical 2- and 3chamber views (Figure 2). The level of the left ventricular papillary muscles marked the division between the mid and distal segments of the LAD. The origin and proximal part of the $\mathrm{Cx}(\mathrm{pCx})$ was found by using the same views as searching for the LM. By focusing on the atrioventricular sulcus, the pCx was seen passing in front of the left atrial appendage. The level of the inferor wall of the left atrial appendage marked the division between the $\mathrm{pCx}$ and middle segment of the $\mathrm{Cx}(\mathrm{mCx})$. By modified parasternal short-axis views, and sometimes subcostal short-axis views, $\mathrm{mCx}$ was visualised as it passed caudally in the atrioventricular sulcus to the inferior margin of the sulcus, and the artery continued further in the atrioventricular sulcus as the distal segment of the $\mathrm{Cx}(\mathrm{dCx})$. The different segments of Cx are imaged in Figure 3 and 4 . With the patient in the right or left decubitus position using parasternal short-axis views, the proximal segment of the RCA (pRCA) was looked for in the area adjacent to the right sinus of Valsalva cranial to the aortic valve (Figure $5 \mathrm{~A})$. The rest of the pRCA was visualised from supine subcostal modified sagittal and 4-chamber views focusing on the anterior tricuspid ring (Figure 5B). The pRCA was considered as the part of RCA passing anterior surface of the tricuspid ring as far as the inferior margin of the right ventricle. Since we had some problems defining the transition 
Table I: Baseline characteristics of the study cohort $(n=I I I)$

\begin{tabular}{|c|c|}
\hline & No of subjects (\%) mean \pm SD \\
\hline Age (years) & $62,9 \pm 9,6$ \\
\hline Heart rate (strokes/minute) & $63 \pm 7,4$ \\
\hline BMI $\left(\mathrm{kg} / \mathrm{m}^{2}\right)$ & $26 \pm 3,6$ \\
\hline Male sex & $82(74,0)$ \\
\hline Total cholesterol (mmol/L) & $4,9 \pm 1,1$ \\
\hline \multicolumn{2}{|l|}{ Blood pressure (mm Hg) } \\
\hline Systolic & $|4| \pm 20$ \\
\hline Diastolic & $82 \pm 12$ \\
\hline \multicolumn{2}{|l|}{ Medical history } \\
\hline Hypertension (> |40/90 mm Hg) & $61(55,0)$ \\
\hline Current smoking & $29(26,1)$ \\
\hline Diabetes & II $(9,9)$ \\
\hline Previous CAD & $24(21,6)$ \\
\hline ACS & $35(3 I, 5)$ \\
\hline \multicolumn{2}{|l|}{ Cardiac medication } \\
\hline Aspirin & $98(88,3)$ \\
\hline Thienopyridine & $38(34,2)$ \\
\hline Low-molecular-weight heparin & $30(27,0)$ \\
\hline$\beta$-Blockers & $87(78,4)$ \\
\hline Statins & $89(80,2)$ \\
\hline Calcium antagonists & $21(18,9)$ \\
\hline ACE-inhibitors/ARB & $25(22,5)$ \\
\hline Organic nitrate, daily maintenance & $13(1 \mid, 7)$ \\
\hline
\end{tabular}

$\mathrm{BMI}=$ body mass index, $\mathrm{CAD}=$ coronary artery disease, $\mathrm{ACS}=$ acute coronary syndrome, $\mathrm{ACE}=$ angiotensin-converting enzyme, $\mathrm{ARB}=$ angiontensin II receptor blocker

between the middle and distal part of the RCA, these segments were labeled together as the middle segment of RCA (mRCA). This combined arterial segment was seen by focusing on the medial and posterior tricuspid ring using subcostal modified short-axis and 4-chamber views (Figure 5C). We labeled the posterior descending artery (PDA) as the distal segment of RCA, and this segment was imaged from modified apical 2-chamber views coursing toward the apex in the posterior interventricular sulcus (Figure 5D).
The coronary artery flow velocity waveform appears as a complex of a small wave in systole and a large trapezoid wave in diastole (Figure 6A). The coronary artery antegrade flow velocities could be low, normal or elevated, and was not always measured. The colour Doppler flow could either be laminar or turbulent. When colour flow Doppler recordings indicated reversed coronary artery flow (the functional diagnosis of coronary obstruction more proximal in the coronary artery), we distinguished the retrograde coronary artery flow from coronary venous 


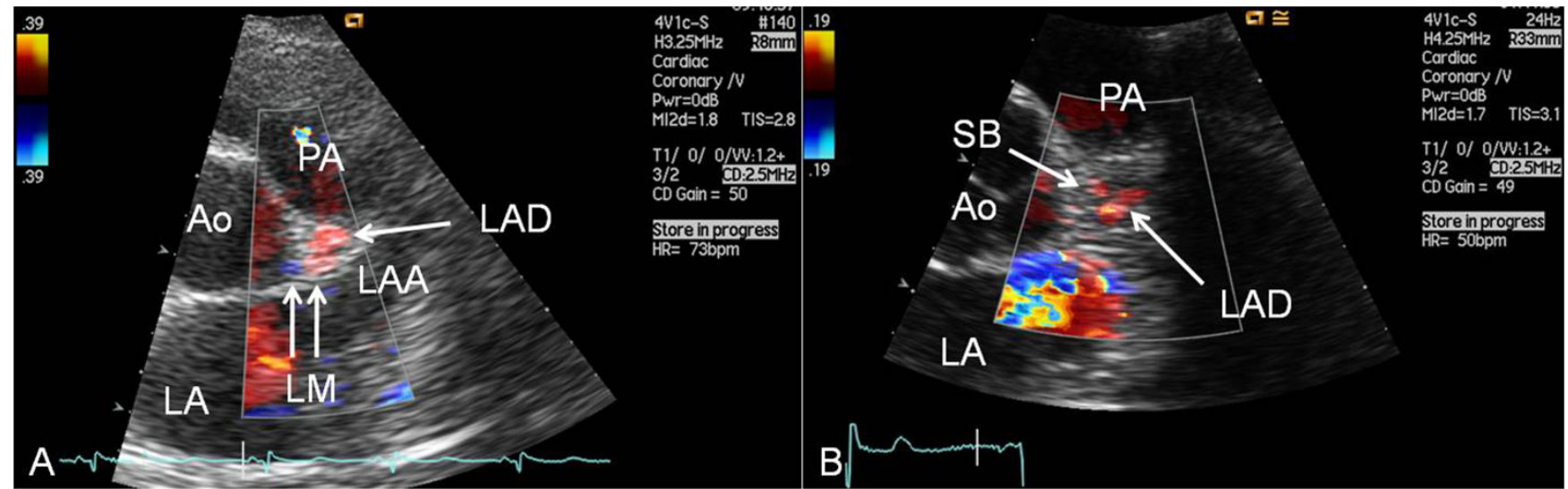

\section{Figure I}

Examples of antegrade coronary artery flow in the LM and proximal parts of LAD. (A) In modified parasternal short-axis view the left main coronary artery (LM) is seen leaving the left sinus of Valsalva and continuing as the left anterior descending coronary artery (LAD) turning slightly towards the transducer. (B) In modified parasternal short-axis view the first septal branch $(S B)$ is seen leaving the LAD. Ao = aortic root; $L A=$ left atrium; LAA = left atrial appendage; PA = pulmonary artery.

flow by finding inverted coronary flow velocity waveform (Figure 6B). In contrast, the coronary venous flow appears as a prominent systolic flow wave.

As outlined above, the LM had one segment and the other main coronary arteries (LAD, Cx and RCA) had each a proximal, middle and distal segment. For each segment three different possibilities were defined: (i) the segment was completely visualised; (ii) the segment was not satisfactorily visualised if any part of the individual segment was not seen or the segment was not visualised at all; (iii) the segment was defined with retrograde flow.

\section{Coronary angiography}

Coronary angiography was performed using standard techniques. All angiograms were classified according to left or right dominance. Collateral flow to occluded arteries was graded according to the Rentrop classification (grade 0 , no visible filling of any collateral channel, grade 1 , filling of side branches of the occluded artery, grade 2, partial filling of the epicardial vessel and grade 3, complete collateral filling of the epicardial vessel) [10]

\section{Statistical analysis}

Continuous variables are presented as mean and standard deviation (SD), or median (5-95 percentiles) if the varia-

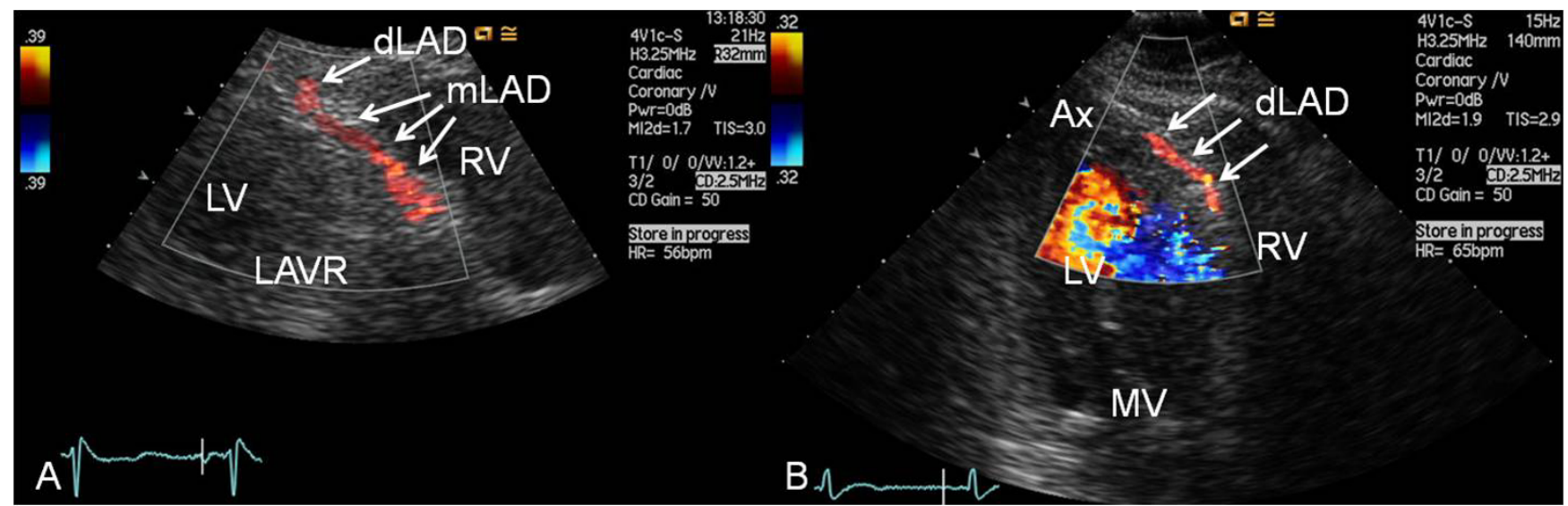

Figure 2

Examples of antegrade coronary artery flow in the middle and distal segments of LAD. (A) The middle left anterior descending coronary artery $(m L A D)$ is imaged from parasternal modified long-axis view focusing on the anterior interventricular sulcus. (B) The distal left anterior descending coronary artery (dLAD) is seen from modified apical 3-chamber view focusing on the anterior interventricular sulcus. $A x=$ apex of the left ventricle; LAVR = left atrioventricular ring; $L V=$ left ventricle; $M V=$ mitral valve; $R V=$ right ventricle. 

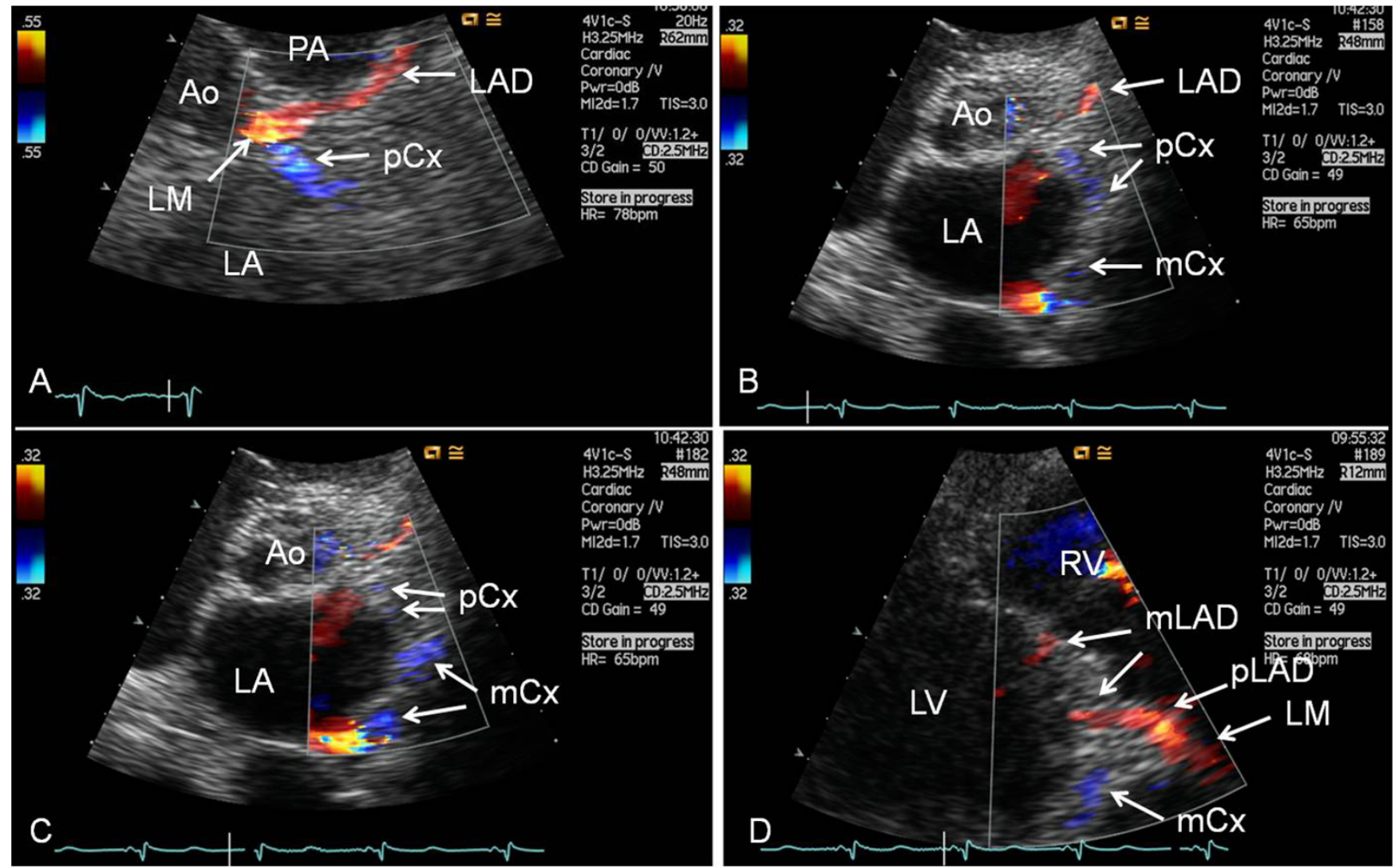

Figure 3

Examples of antegrade coronary artery flow in the proximal and middle segments of Cx. (A) In modified parasternal short-axis view focusing on the area adjacent to the left sinus of Valsalva the proximal circumflex coronary artery ( $\mathrm{pCx}$ ) is seen leaving the left main coronary artery (LM). ( $B$ and $C$ ) In modified parasternal short-axis views the proximal and middle segments of the circumflex coronary artery (Cx) are found passing caudally in the lateral atrioventricular sulcus. (D) From parasternal modified long-axis view focusing on the lateral atrioventricular sulcus the middle $\mathrm{Cx}(\mathrm{mCx})$ is seen traversing caudally. $A_{o}=$ aortic root/valve; $L A=$ left atrium; $L A D=$ left anterior descending coronary artery; $L V=$ left ventricle; $m L A D=$ middle segment of LAD; PA = pulmonary artery; $P L A D=$ proximal segment of $L A D ; R V=$ right ventricle.

bles were skewed. Categorical variables are presented as fractions/percent. Univariate regression analyses were used to explore relationships between success rate and demographic and clinical variables. Variables in univariate testing with $\mathrm{p}<1.10$ were entered in multivariate forward analyses. The success rates among different patient sub-populations were compared using the unconditional z-pooled test for binomial proportions, with confidence interval method with coefficient 0.9999 . $\mathrm{P}<0.05$ was considered statistically significant. All analyses were done with SPSS for windows (SPSS Inc, Chicago, IL, v 15,0).

\section{Results}

Visualisation of different coronary artery segments Left main coronary artery

Complete imaging of the LM was achieved in all but two patients (98\%) (Table 2).
Left anterior descending artery

Adding the patients with completely seen segments with antegrade coronary flow and patients with segmental retrograde coronary flow, the proximal, middle and distal segments of LAD were adequately visualised in $96 \%, 96 \%$ and $93 \%$ of the patients, respectively (Table 2 ). With antegrade directed coronary artery flow, the proximal, middle and distal segments of LAD were entirely seen in $96 \%$, $95 \%$ and $91 \%$ of the patients, respectively (Table 2). Retrograde coronary artery flow was seen in one middle and two distal segments of the LAD (Table 2). Coronary angiography demonstrated total occlusion of LAD in these cases with collateral flow to the occluded artery in Rentrop class $\geq 2$. Visualisation of both LM and PLAD or LM and pLAD/mLAD was achieved in $95 \%$ and $90 \%$ of the patients (Table 3). Visualisation of the LM together with all segments of LAD was achieved in $85 \%$ of patients (Table 3 ). The visualisation of LM and the complete LAD 


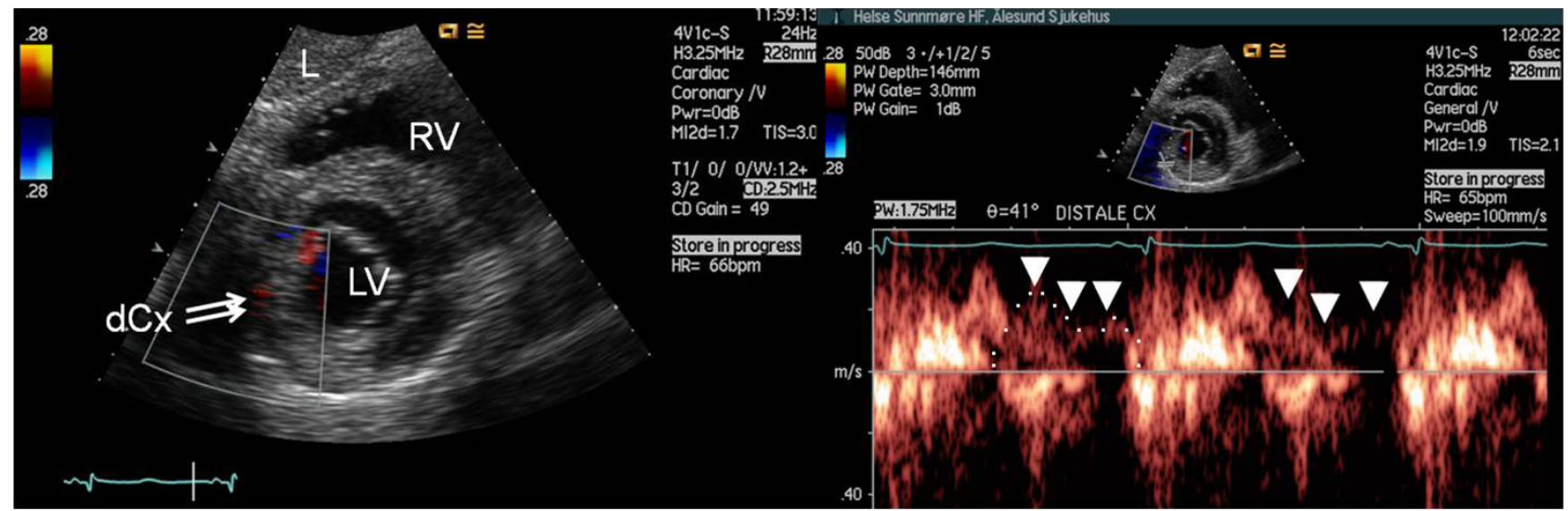

Figure 4

Example of antegrade coronary artery flow in the distal segment of Cx. The distal segment of the circumflex coronary artery $(\mathrm{dCx})$ imaged by colour Doppler mapping and matching spectral Doppler tracings of blood flow (with arrowheads denoting diastolic flow waveform with one diastolic flow velocity waveform enveloped), imaged from modified subcostal shortaxis view focusing on the inferior atrioventricular sulcus. $L=$ liver; $L V=$ left ventricle; $R V=$ right ventricle.

was negatively related to patients' body mass index (BMI) $(\mathrm{p}=0.002)$ in the regression analyses.

\section{Circumflex coronary artery}

With antegrade directed coronary artery flow, the proximal, middle and distal segments of Cx were completely seen in $88 \%, 61 \%$ and $3 \%$ of the patients, respectively (Table 2). No patient was found to have retrograde flow. Both pCx and mCx could be visualised in $61 \%$ of patients, while all three segments of $\mathrm{Cx}$ could be seen in 3\% of patients (Table 3). Visualisation of the complete $\mathrm{Cx}$ or the proximal and mid segments was negatively related to patient age $(p=0,03)$ in univariate regression analyses. Visualisation of the complete $\mathrm{Cx}$ was negatively related to age $(\mathrm{p}=0,01)$ and heart rate $(\mathrm{p}=0,03)$ in multivariate analyses.

\section{Right coronary artery}

Adding the patients with completely seen segments with antegrade coronary flow and patients with segmental retrograde coronary flow, the PRCA, mRCA and PDA were adequately visualised in $40 \%, 29 \%$ and $59 \%$, respectively (Table 2). With antegrade directed coronary artery flow, the proximal and middle segments of the RCA and PDA were entirely seen in $40 \%, 28 \%$ and $54 \%$, respectively (Table 2). Retrograde coronary artery flow was seen in one mRCA and five PDAs (Table 2). Coronary angiography showed corresponding findings apart from two PDAs which had antegrade flow on angiography. In the cases where retrograde Doppler flow in PDA was confirmed angiographically collateral circulation in PDA was Rentrop class $\geq 2$. Both the PRCA and PDA were visualised in $27 \%$ of patients, while the total RCA/PDA was adequately imaged in $15 \%$ of patients (Table 3 ). Visualisation of the complete RCA/PDA was negatively related to patient BMI in regression analyses $(\mathrm{p}=0.01)$.

Visualisation of proximal versus distal coronary segments Visualisation of all segments of the coronary arteries was achieved in only one patient (1\%) (Table 3). There were significant differences in the visualisation of proximal versus distal segments between the different coronary arteries. In contrast with $\mathrm{Cx}$ and RCA, the rate of visualisation of distal and proximal segments of LAD was equal (Table 2). In $C x$ the distal segment was visualised in only a minority of patients. In RCA it was the middle segment which was most difficult to visualise (Table 2). The LM and proximal segments of LAD, Cx and RCA were visualised in 35\% of patients. Excluding RCA, LM and proximal segments of LAD and Cx were adequately seen in $85 \%$ of patients (Table 3).

\section{Right versus left coronary dominance}

Coronary angiograms were available for 108 patients, and 8 patients were found to have dominant $\mathrm{Cx}$, supplying the PDA from the distal continuation of the $\mathrm{Cx}$. The remaining patients had the RCA as the dominant artery, supplying the PDA. Among the eight patients with left coronary dominance, the $\mathrm{pCx}, \mathrm{mCx}$ and $\mathrm{dCx}$ were completely visualised in seven, six and one patients, respectively. Among the same patients, the pRCA, mRCA and PDA were completely visualised in zero, one and two patients, respectively. The statistical analyses showed a trend towards lower feasibility of adequate visualisation of pRCA and mRCA and a trend towards higher feasibility of visualisation of $\mathrm{pCx}$ and $\mathrm{mCx}$ when the $\mathrm{Cx}$ was the dominant coronary artery. 


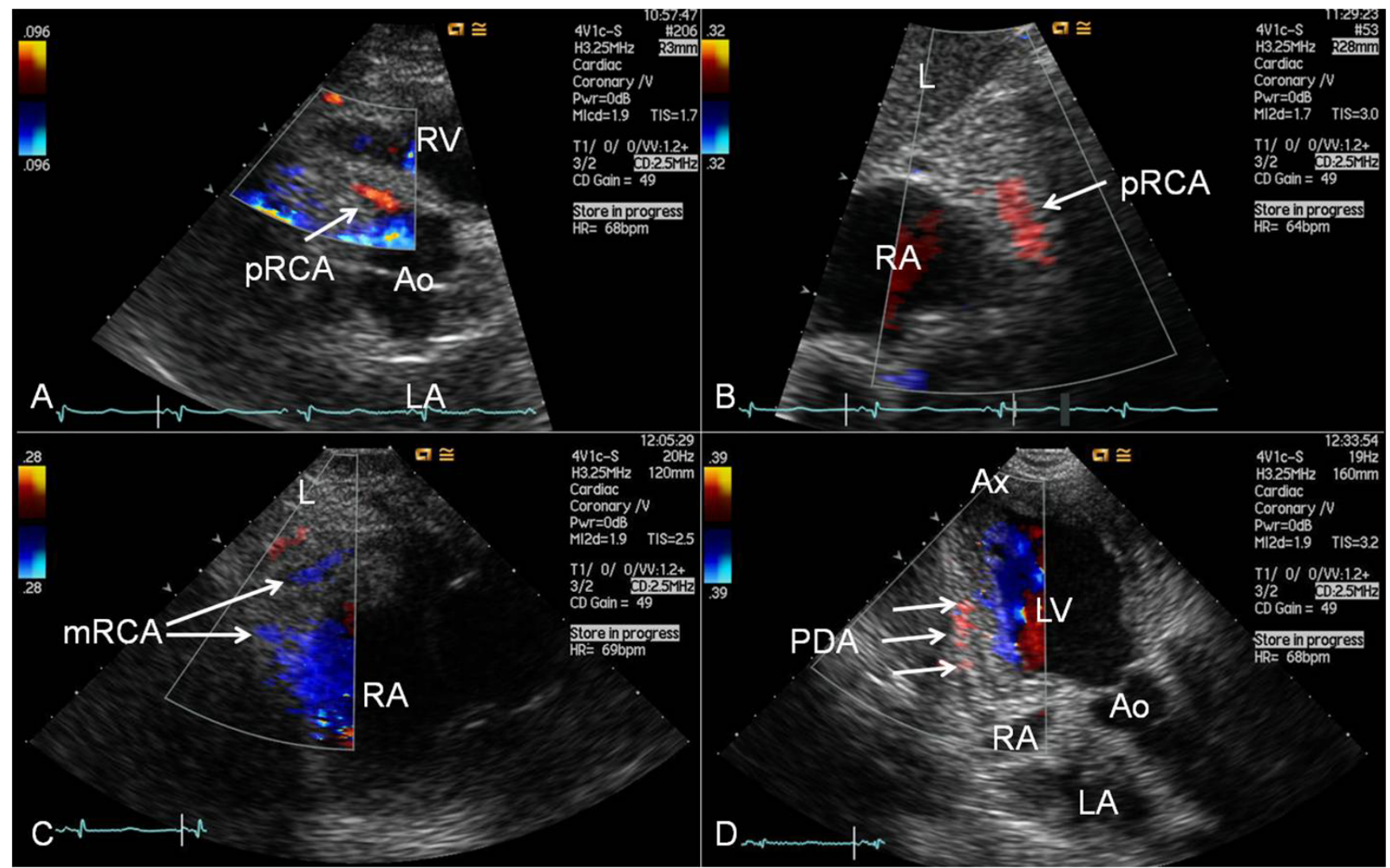

\section{Figure 5}

Examples of antegrade coronary artery flow in the RCA and PDA. (A) In modified parasternal short-axis view searching the area adjacent to the right sinus of Valsalva the proximal right coronary artery ( $\mathrm{PCA}$ ) is seen leaving the aortic root (Ao). (B) From subcostal modified sagittal view the pRCA is seen traversing caudally on the anterior tricuspid ring. (C) Using subcostal modified short-axis view parts of the middle segment of the right coronary artery (mRCA) are seen coursing medially on the medial tricuspid ring. (D) From modified apical 2-chamber view focusing on the posterior interventricular sulcus parts of the posterior descending coronary artery (PDA) are seen coursing toward the apex $(A x)$. $L=$ liver; $L A=$ left atrium; $\mathrm{LV}=$ left ventricle; $\mathrm{RA}=$ right atrium; $\mathrm{RV}=$ right ventricle.

\section{Visualisation of different coronary artery segments according to clinical presentation}

Statistical analyses showed no significant differences in the degree of adequate visualisation of the various main coronary segments when comparing patients with or without acute coronary syndrome, or comparing patients with or without known coronary disease.

\section{Discussion}

In this study of consecutively included patients with suspected or definite coronary artery disease the feasibility of transthoracic echocardiography for visualisation of different segments of the main coronary arteries was examined. The purpose of the study was to elucidate the feasibility of viewing the complete coronary artery segments with antegrade flow or findings of retrograde coronary artery flow. The main finding was that the left main, the LAD segments and the proximal Cx segment could be completely imaged in the great majority of patients while the mid and distal Cx segments and the RCA were less well imaged.
Use of TTE for direct inspection of various parts of the main coronary arteries has been the subject of several studies, both for detection of coronary stenoses and occlusions $[2,3,3-9,11-15]$. Coronary occlusions may be detected by retrograde flow in the coronary artery [6-9]. Coronary stenoses can be identified by local flow acceleration and turbulence expressed as colour aliasing by colour flow Doppler and accelerated flow velocities across the stenosis, with further quantification of the individual stenosis by comparing flow velocities at the site of aliasing with nearest upstream non-accelerated prestenotic flow velocities $[2-5,11,12]$. A stenotic to prestenotic flow velocity ratio (SPVR) exceeding 2 has been proposed as a cutoff value for significant stenosis [2,3]. Accelerated coronary artery flow velocities typically give a stronger colour Doppler signal than normal laminar coronary flow, and these turbulent flow signals may be detected without visualisation of adjacent up- or downstream coronary portions [2]. However, entire imaging of the individual coronary segment should ease the demonstration of pos- 

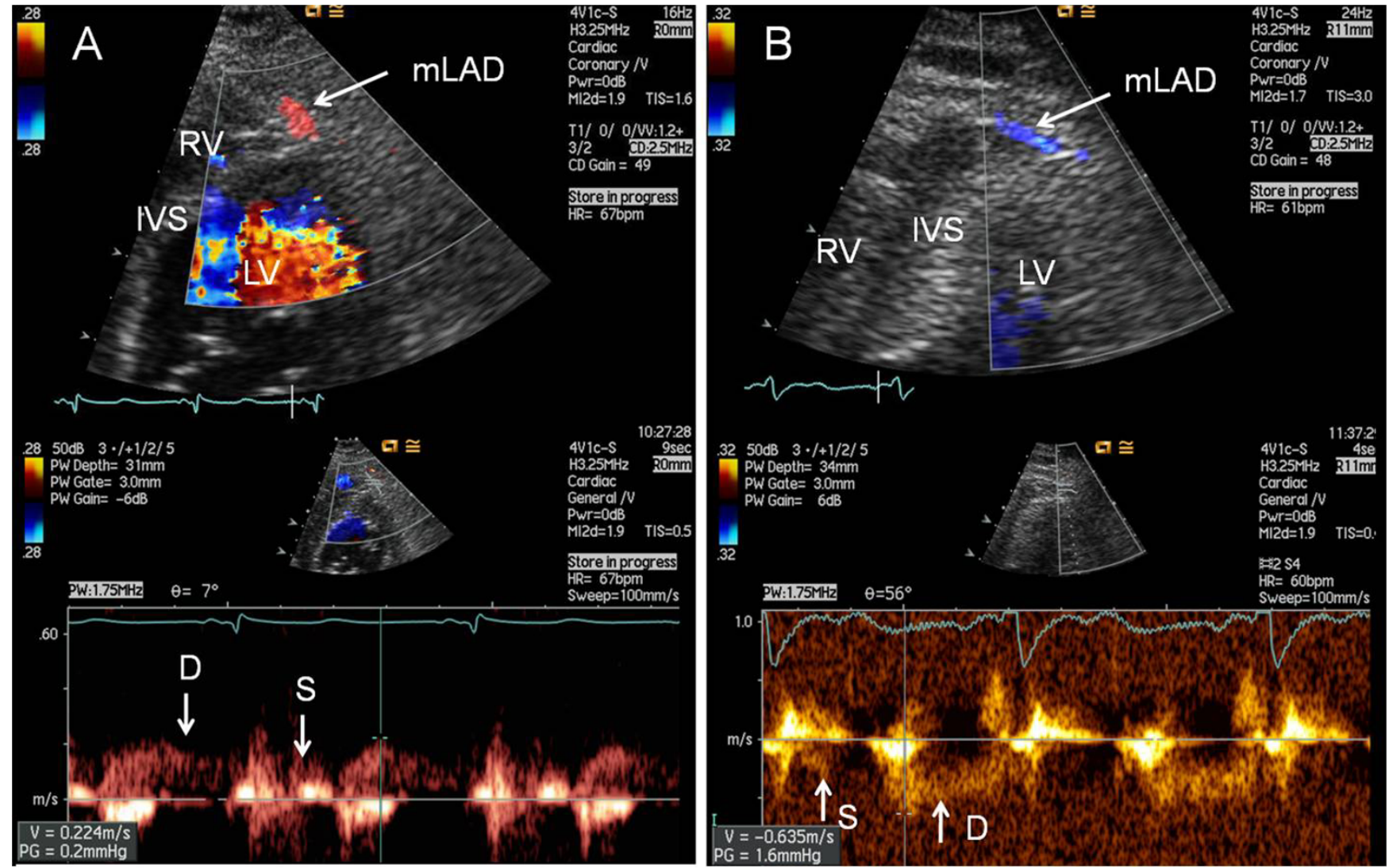

Figure 6

Examples of antegrade and retrograde flow in the middle segment of LAD. The middle segment of the left anterior descending coronary artery (mLAD) imaged by colour Doppler mapping and matching spectral Doppler tracings of blood flow, imaged from modified parasternal short-axis view focusing on the anterior interventricular sulcus: (A) The mLAD is seen with antegrade flow. (B) The $m L A D$ is seen with retrograde flow. $D=$ spectral Doppler tracings of diastolic coronary blood flow; IVS = interventricular septum; LV = left ventricle; $R V$ = right ventricle; $S$ = spectral Doppler tracings of systolic coronary blood flow.

sible stenoses with further Doppler measurements of flow velocities. So far, the largest study identifying stenoses by searching the various main coronary artery segments for flow turbulence and a stenotic SPVR included 84 patients, without performing detailed analyses on the visibility of the different coronary artery segments by TTE [2]. In contrast with previous studies, our study tried to elucidate the feasibility of complete visualisation of the various main coronary artery segments, in a larger set of patients with various degrees of atherosclerotic coronary disease.

Findings of retrograde flow in the main coronary arteries by TTE have high accuracy in documenting LAD occlusions [6-8] and RCA/PDA occlusions [7,9]. Retrograde coronary artery flow was rarely found in our patient cohort; in only nine main coronary artery segments, mainly in the PDA and dLAD (Table 2). Our findings of retrograde flow by TTE corresponded well with findings by coronary angiography, with matching findings in seven of nine patients. In the two patients falsely found with ret- rograde flow in the PDA by TTE, we suspect that the distal part of PDA was confused with the recurrent branch of the dLAD running around the apex of the heart.

Although large portions of any coronary artery segment with antegrade flow often could be satisfactorily seen in our study, the whole segment (with antegrade flow) had to be imaged to be labeled completely visualised, as outlined above. Complete visualisation of the various main coronary artery segments was dependent on both the artery and segment investigated (Table 2). Our study demonstrates that TTE is a feasible method for complete visualisation of different coronary artery segments with antegrade flow, especially the LM, the proximal segments of LAD and $\mathrm{Cx}$, and the middle and distal segments of LAD. The distal Cx and the RCA segments were not adequately visualised. This may be due to several factors including individual variations in coronary anatomy for the $\mathrm{Cx}$ and the RCA. Even with use of invasive coronary angiography the distal segments of $\mathrm{Cx}$ may be difficult to 
Table 2: Findings of visualisation of individual coronary artery segments

\begin{tabular}{|c|c|c|c|}
\hline Segment & $\begin{array}{c}\text { Antegrade flow or retrograde flow } \\
n(\%)\end{array}$ & $\begin{array}{c}\text { Antegrade flow } \\
\text { n (\%) }\end{array}$ & $\begin{array}{l}\text { Retrograde flow } \\
\text { n (\%) }\end{array}$ \\
\hline LM & $109(98,2)$ & $109(98,2)$ & $0(0)$ \\
\hline pLAD & $106(95,5)$ & $106(95,5)$ & $0(0)$ \\
\hline mLAD & $106(95,5)$ & $105(94,6)$ & I $(0,9)$ \\
\hline dLAD & $103(92,8)$ & $101(91,0)$ & $2(1,8)$ \\
\hline$p C x$ & $98(88,3)$ & $98(88,3)$ & $0(0)$ \\
\hline $\mathrm{mCx}$ & $68(61,3)$ & $68(61,3)$ & $0(0)$ \\
\hline$d C x$ & $3(2,7)$ & $3(2,7)$ & $0(0)$ \\
\hline $\mathrm{pRCA}$ & $44(39,6)$ & $44(39,6)$ & $0(0)$ \\
\hline $\mathrm{mRCA}$ & $32(28,8)$ & $31(27,9)$ & I $(0,9)$ \\
\hline PDA & $65(58,6)$ & $60(54,1)$ & $5(4,5)$ \\
\hline
\end{tabular}

Findings of complete visualisation of coronary artery segments with antegrade flow or coronary segments found with retrograde flow $(\mathrm{n}=\mathrm{I} \mathrm{I} \mathrm{I})$. LM $=$ left main coronary artery, $\mathrm{PLAD}=$ proximal segment of left anterior descending artery, $\mathrm{mLAD}=$ middle segment of left anterior descending artery, $d L A D=$ distal segment of left anterior descending artery, $\mathrm{pCx}=$ proximal segment of circumflex coronary artery, $\mathrm{mCx}=$ middle segment of circumflex coronary artery, $\mathrm{dCx}=$ distal segment of circumflex coronary artery, $\mathrm{PRCA}=$ proximal segment of right coronary artery, $\mathrm{mRCA}=$ middle segment of right coronary artery, PDA = posterior descending artery

define in some patients. The small study group with left coronary dominance showed a trend towards more complete visualisation of the proximal and mid segments of $\mathrm{Cx}$ and of less adequate visualisation of pRCA and mRCA. Patients' BMI was a significant predictor of success rate in our study. Our results are indicative of what can be expected with this technique in the hands of experienced echocardiographers and currently available equipment. Further developments of echocardiographic technology may both increase the feasibility of visualisation of coronary arteries by TTE and diminish echocardiographic limitations caused by overweight.

Earlier studies using coronary colour flow Doppler TTE are not directly comparable to our study, since those studies primarily focused on searching for coronary artery stenoses and occlusions [2-4,6-9,11-14], without fully describing the degree of completeness of imaging of the coronary artery segment investigated. Several of these studies only concentrated on the LM and proximal and mid segments of LAD $[6,8,11-14]$. Studies focusing on coronary stenoses by use of coronary flow turbulence and stenotic SPVR have reported high accuracy for detecting stenotic lesions in the $\mathrm{LM}$ and $\mathrm{LAD}$, but lower precision for detection of stenoses in the Cx and RCA/RDP [2-4,12]. Incomplete imaging of the individual coronary segments could explain failure of identification of stenotic coronary flow turbulence.

\section{Limitations of the study}

There are several limitations to our study. Use of intravenous ultrasound contrast agent might have improved the results. Because of limited clinical experience in patients with acute coronary syndrome, we chose not to use ultrasound contrast when planning the study. We can not exclude the possibility of overstating the completeness of

Table 3: Visualisation of several coronary artery segments combined

\begin{tabular}{|c|c|}
\hline & No of subjects (\%) \\
\hline$L M+p L A D+m L A D+d L A D$ & $94(84,7)$ \\
\hline$L M+p L A D$ & $105(94,6)$ \\
\hline$L M+p L A D+m L A D$ & $100(90,1)$ \\
\hline$p C x+m C x+d C x$ & $3(2,7)$ \\
\hline$p C x+m C X$ & $68(61,3)$ \\
\hline $\mathrm{pRCA}+\mathrm{mRCA}+\mathrm{PDA}$ & $17(15,3)$ \\
\hline $\mathrm{PRCA}+\mathrm{PDA}$ & $30(27,0)$ \\
\hline$L M+$ all segments of $L A D / C X / R C A$ & I $(0,9)$ \\
\hline$L M+p L A D+m L A D+p C x+m C x+p R C A+m R C A$ & $14(12,6)$ \\
\hline$L M+p L A D+p C x+p R C A$ & $39(35,1)$ \\
\hline$L M+p L A D+p C x$ & $94(84,7)$ \\
\hline
\end{tabular}

Visualisation of several coronary artery segments combined, with each individual coronary segment either completely imaged with antegrade flow or found with retrograde flow $(n=I I I)$. $L M=$ left main coronary artery, $\mathrm{PLAD}=$ proximal segment of left anterior descending artery, $\mathrm{mLAD}=$ middle segment of left anterior descending artery, $d L A D=$ distal segment of left anterior descending artery, $p C x=$ proximal segment of circumflex coronary artery, $\mathrm{mCx}=$ middle segment of circumflex coronary artery, $\mathrm{dCx}=$ distal segment of circumflex coronary artery, $\mathrm{pRCA}=$ proximal segment of right coronary artery, $\mathrm{mRCA}=$ middle segment or right coronary artery, PDA = posterior descending artery 
imaging of the individual coronary artery segments. However, when changing the echocardiographic view we always tried to find overlapping end-portions of the coronary artery compared with the foregoing views, and alternative views were used to fill in any missing portions of the individual coronary artery segment. Moreover, we can not exclude that nearby parallel coursing coronary artery branches occasionally may have been mistaken as the main coronary segment of the dLAD or PDA. In addition, retrograde flow of the distal part of PDA may have been confused with the recurrent branch of the dLAD, which runs around the apex of the heart. Finally, we can not exclude selection bias in our results, since our study cohort only included patients planned for coronary angiography and excluded patients with previous coronary artery bypass surgery, presumed insufficient acoustic windows, significant valvular disease or atrial fibrillation.

\section{Conclusions}

Complete visualisation of the various main coronary artery segments is dependent on both the artery and segment investigated. TTE is a feasible method for complete demonstration of antegrade coronary flow in the LM, the proximal $\mathrm{Cx}$ and the different segments of LAD, but less suitable for RCA and mid and distal segments of Cx. Complete visualisation of individual coronary segments might ease the demonstration of coronary stenoses by TTE. Detection of segmental retrograde coronary flow by TTE corresponds well with coronary angiography.

\section{Competing interests}

The authors declare that they have no competing interests.

\section{Authors' contributions}

JV conceived the study, carried out the ultrasound examinations and drafted the manuscript, EH carried out the ultrasound examinations and drafted the manuscript, RW carried out the angiography readings and helped to draft the manuscript, KH carried out the angiography readings, TH participated in the design of the study, performed the statistical analyses and helped to draft the manuscript. All the authors read and approved the final manuscript.

\section{Acknowledgements}

The study was funded by grants from Sunnmore Health Trust Research Fund and Helse Midt-Norge Regional Health Trust Research Fund.

\section{References}

I. Krzanowski M, Bodzo• W, Dimitrow PP: Imaging of all three coronary arteries by artery transthoracic echocardiography. An illustrated guide. Cardiovascular Ultrasound 2003, I: I6.

2. Saraste M, Vesalainen RK, Ylitalo A, Saraste A, Koskenvuo JW, Toikka JO, Vaittinen M-A, Hartiala JJ, Airaksinen KEJ: Transthoracic Doppler echocardiography as a non-invasive tool to assess coronary artery stenoses - a comparison with quantitative coronary angiography. J Am Soc Echocardiogr 2005, I 8:679-85.

3. Krzanowski M, Bodzo• W, Dudek D, Heba G, Rzeszutko M, Nieankowski R, Dubiel J, Szczeklik A: Transthoracic, harmonic mode, contrast enhanced color Doppler echocardiography in detection of restenosis after percutaneous coronary interventions. Prospective evaluation verified by coronary angiography. Eur J Echocardiography 2004, 5:5I-64.

4. Krzanowski M, Bodzo• W, Brzostek T, Ni॰ankowski R, Szczeklik A: Value of transthoracic echocardiography for the detection of high-grade coronary artery stenosis: prospective evaluation in $\mathbf{5 0}$ consecutive patients scheduled for coronary angiography. J Am Soc Echocardiogr 2000, I3:1091-9.

5. Holte $E$, Vegsundvåg J, Wiseth R: Direct visualisation of a significant stenosis of the right coronary artery by transthoracic echocardiography. A case report. Cardiovascular Ultrasound 2007, 5:33.

6. Hirata K, Watanabe H, Hozumi T, Tokai K, Otsuka R, Fujimoto K, Shimada K, Muro T, Yoshiyama M, Yoshikawa J: Simple detection of occluded coronary artery using retrograde flow in septal branch and left anterior descending coronary artery by transthoracic Doppler echocardiography at rest. J Am Soc Echocardiogr 2004, I 7:108-13.

7. Boshchenko AA, Vrublevsky AV, Karpov RS: Transthoracic echocardiography in the detection of chronic total coronary artery occlusion. Eur J Echocardiography 2009, 1 0:62-68.

8. Watanabe N, Akasaka T, Yamaura Y, Akiyama M, Koyama Y, Kamiyama N, Neishi Y, Kaji S, Saito Y, Yoshida K: Noninvasive detection of total occlusion of the left anterior descending coronary artery with transthoracic Doppler echocardiography. J Am Coll Cardiol 200I, 38:1328-32.

9. Otsuka R, Watanabe H, Hirata K, Tokai K, Muro T, Hozumi T, Yoshiyama M, Yoshikawa J: A novel technique to detect total occlusion in the right coronary artery using retrograde flow by transthoracic Doppler echocardiography. J Am Soc Echocardiogr 2005, I 8:704-9.

10. Rentrop KP, Cohen M, Blanke H, Phillips RA: Changes in collateral channel filling immediately after controlled coronary artery occlusion by an angioplasty balloon in human subjects. J Am Coll Cardiol 1985, 5:587-92.

II. Hozumi T, Yoshida K, Akasaka T, Asami Y, Kanzaki Y, Ueda Y, Yamamuro A, Takagi T, Yoshikawa J: Value of acceleration flow and the prestenotic to stenotic coronary flow velocity ratio by transthoracic color Doppler echocardiography in noninvasive diagnosis of restenosis after percutaneous transluminal coronary angioplasty. J Am Coll Cardiol 2000, 35: 164-8.

12. Saraste M, Koskenvuo JW, Mikkola J, Pelttari L, Toikka JO, Hartiala JJ: Technical achievement: transthoracic Doppler echocardiography can be used to detect LAD restenosis after coronary angioplasty. Clin Physiol 2000, 6:428-33.

13. Anjaneyulu A, Raghavaraju P, Krishnaswamy R, Johann C, Krishnamraju $P$, Rajagopalaraju A, Somaraju B: Demonstration of recanalized left coronary artery after thrombolysis by transthoracic echocardiography. J Am Soc Echocardiogr 2005, I 8:686-92.

14. Anjaneyulu A, Raghu K, Chandramukhi S, Satyajit GM, Arramraja S, Raghavaraju P, Krishnamraju P, Somaraju B: Evaluation of left main coronary artery stenosis by transthoracic echocardiography. J Am Soc Echocardiogr 2008, 2 I:855-60.

15. Caiati C, Zedda N, Cadeddu M, Chen L, Montaldo C, Iliceto S, Lepera $\mathrm{ME}$, Favale S: Detection, location, and severity assessment of left anterior descending coronary artery stenoses by means of contrast-enhanced transthoracic harmonic echo Doppler. Eur Heart J 2009, 30:1797-806.

Publish with Biomed Central and every scientist can read your work free of charge

"BioMed Central will be the most significant development for disseminating the results of biomedical research in our lifetime. "

Sir Paul Nurse, Cancer Research UK

Your research papers will be:

- available free of charge to the entire biomedical community

- peer reviewed and published immediately upon acceptance

- cited in PubMed and archived on PubMed Central

- yours - you keep the copyright
BiolMedcentral 